Divane Leite Matos 1,2

\title{
Projeto Bambuí: estudo de base populacional dos fatores associados com o uso regular de serviços odontológicos em adultos
}

Maria Fernanda F. Lima-Costa 1,2

Henrique L. Guerra ${ }^{2}$

Wagner Marcenes 3,4

\author{
The Bambuí Project: a population-based \\ study of factors associated with regular \\ dental care in adults
}

\footnotetext{
1 Departamento de Medicina Preventiva e Social,

Faculdade de Medicina Universidade Federal de Minas Gerais. Av. Alfredo Balena 190 Belo Horizonte, $M G$ 30130-100, Brasil.

2 Laboratório de

Epidemiologia e Antropologia Médica, Centro de Pesquisas René Rachou, Fundação Oswaldo Cruz. Av. Augusto de Lima 1715, Belo Horizonte, $M G$ 30190-002, Brasil.

3 Department of Epidemiology and Public Health, Royal Free and University College Medical School.

1-19 Torrington Place, London WC1E 6BT, UK

${ }^{4}$ Department of Adult Oral Health, St' Bartholomew's and the Royal London School of Medicine and Dentistry. Turner Street, London E1 $2 A D, U K$
}

\begin{abstract}
A cross-sectional study was conducted in Bambuí, Minas Gerais, to identify factors associated with regular use of dental services. Participants were interviewed with a structured questionnaire and previously validated questions. 999/1,221 (81.8\%) randomly selected individuals aged $>18$ years participated in the Bambui dental survey. Of these, 654 out of 656 individuals who had at least one natural tooth and had visited a dentist during their lifetime participated in the study. Results adjusted by multiple logistic regression showed that regular use of dental services was significantly related to having $>8$ and 4-7 years of schooling $(O R=9.90 ; 95 \% C I=$ 2.90-33.77 and $O R=3.87 ; 95 \% C I=1.11-13.51$, respectively), having a preference for restorative treatment rather than extraction $(O R=4.91 ; 95 \% C I=2.23-10.79)$, having no present need of dental treatment $(O R=4.87 ; 95 \% C I=3.17-7.49)$, and belief that visiting the dentist prevents tooth decay and gum disease $(O R=1.73 ; 95 \% C I=1.13-2.65)$. The results show that regular use of dental services was related to factors distributed in the Andersen and Newman model (1973) explaining use of dentistry services.
\end{abstract}

Key words Dentistry; Dental Care; Adult Health

Resumo Um estudo seccional foi desenvolvido na cidade de Bambuí, Minas Gerais, com o objetivo de identificar fatores associados ao uso regular de serviços odontológicos entre adultos. Os participantes foram entrevistados utilizando-se um questionário estruturado e questões previamente validadas. Um total de 999/1221 (81,8\%) indivíduos com idade $\geq 18$ anos selecionados aleatoriamente participaram do inquérito de saúde bucal. Destes, 654 entre 656 indivíduos que possuíam pelo menos um dente natural e haviam visitado o dentista pelo menos uma vez na vida participaram do presente trabalho. Resultados ajustados pela regressão logística múltipla mostraram que o uso regular de serviços odontológicos foi significativamente associado a ter $\geq 8$ e 4-7 anos de escolaridade (OR = 9,90; IC 95\% = 2,90-33,77 e OR = 3,87; IC 95\% = 1,11-13,51, respectivamente), ter preferência para tratamento dentário restaurador em comparação à extração $(O R=4,91 ;$ IC 95\% = 2,23-10,79), não ter necessidade atual de tratamento dentário $(O R=4,87 ;$ IC $95 \%=3,17-7,49)$ e acreditar que ir ao dentista previne a cárie e a doença na gengiva $(O R=$ 1,73; IC 95\% = 1,13-2,65). Os resultados mostram que o uso regular de serviços odontológicos na população estudada foi explicado nas diferentes dimensões do modelo elaborado por Andersen \& Newman (1973).

Palavras-chave Odontologia; Assistência Odontológica; Saúde do Adulto 


\section{Introdução}

Várias abordagens têm sido propostas (Andersen \& Newman, 1973; Grembowski et al., 1989; Petersen \& Pedersen, 1984) para melhor conceituar e operacionalizar as investigações sobre o uso de serviços odontológicos. Abordagem amplamente utilizada é a proposta por Andersen \& Newman (1973), cujo modelo foi utilizado inicialmente para estudar os determinantes do uso de serviços médicos. Porém, mais recentemente, tem sido também utilizado em estudos sobre o uso de serviços odontológicos. Três tipos de características são consideradas para explicar as diferenças no uso de serviços de saúde neste modelo: predisposição, facilitação e necessidade. No primeiro tipo, representando a predisposição do indivíduo para usar os serviços de saúde, incluem-se variáveis sócio-demográficas (idade, sexo, estado civil, tamanho da família, etnia, escolaridade e ocupação) e atitudes e opiniões do indivíduo (valores em relação à saúde e conhecimento sobre a doença). No segundo tipo incluem-se variáveis que facilitam o uso desses serviços (renda familiar, existência ou não de plano de saúde e acesso à fonte regular de cuidado dentário). O terceiro tipo, representando a necessidade do uso de serviços de saúde, inclui a percepção do indivíduo ou o diagnóstico da doença avaliados pelo profissional de saúde.

Diversos trabalhos realizados em países desenvolvidos utilizaram o modelo proposto por Andersen \& Newman (1973) para explicar o uso de serviços odontológicos (Gilbert et al., 1993, 1997; Locker \& Clarke, 1999; Ryan et al., 1996; Swank et al., 1986; Tennstedt et al., 1994). Os resultados desses e de outros estudos (Brown \& Lazar, 1999; Douglass \& Cole, 1979; Honkala et al., 1997; Schwarz, 1996) mostram de forma consistente que pessoas do sexo feminino, aquelas com idade entre 5 a 24 anos e pessoas com maior renda e escolaridade utilizam os serviços odontológicos com mais freqüência e/ou tendem a usá-los de forma regular. O uso de serviços odontológicos tem sido também associado à percepção da necessidade de tratamento pelo indivíduo (Cherry-Peppers et al., 1995; Chisick et al., 1997; Tennstedt et al., 1994).

Outras variáveis que poderiam ser importantes para explicar o uso de serviços odontológicos, mas ainda pouco estudadas, são o valor que se dá ao dente (tratamento preferido) e a crença de que ir ao dentista evita as doenças bucais (Gilbert et al., 1997).

Estudos dos fatores associados ao uso de serviços odontológicos são raros no Brasil (Cesar et al., 1995). No presente trabalho são ana- lisados os fatores associados ao uso regular de serviços odontológicos em uma cidade do interior do país, examinando a influência de variáveis de predisposição, facilitação e necessidade de tratamento odontológico.

\section{Material e métodos}

\section{Projeto Bambuí}

O Projeto Bambuí é um estudo com dois componentes: o primeiro, é um estudo prospectivo com base populacional da população idosa (sessenta ou mais anos); o segundo, é um inquérito de saúde da população com cinco ou mais anos de idade (Lima-Costa et al., 2000). O presente trabalho é parte deste inquérito de saúde.

\section{Área estudada}

O Município de Bambuí (21.143 habitantes) situa-se no oeste de Minas Gerais, a cerca de 260 $\mathrm{km}$ de Belo Horizonte. Este trabalho foi desenvolvido na sede do município onde se concentram cerca de $70 \%$ dos moradores do mesmo (IBGE, 1992). A atividade econômica de Bambuí consiste, basicamente, na agricultura, na pecuária leiteira e no comércio (Prefeitura Municipal de Bambuí, 1997).

Em seu sistema de saúde, o município possui uma policlínica municipal para atendimento ambulatorial e um hospital geral. Em relação à atenção odontológica, há uma forte predominância do setor privado (18 cirurgiõesdentistas) e uma tímida intervenção do setor público, prevalecendo uma prática eminentemente curativa. O atendimento odontológico pelo sistema público, financiado pelo Sistema Único de Saúde (SUS), está distribuído entre três escolas de 1o Grau, onde os estudantes e a população adulta são atendidos de forma não sistematizada e não organizada. A equipe é formada por cinco cirurgiões-dentistas e uma auxiliar de consultório dentário. O tipo de tratamento oferecido é simples, sendo realizadas extrações dentárias, restaurações e limpeza dos dentes. A taxa dentista/população é igual a 1 por 1.175 habitantes, refletindo o que ocorre no Brasil (1/1.007) e sendo razoavelmente semelhante à taxa de países desenvolvidos como os Estados Unidos (1/1.598) e o Canadá (1/ 1.873) (Marcos, 2000). 


\section{População estudada}

Foi realizado um censo da cidade de Bambuí entre outubro e novembro de 1994 para a identificação da população base do inquérito de saúde. Uma amostra probabilística simples sem reposição de 1664 moradores com idade igual a cinco ou mais anos foi selecionada para participar deste inquérito. Maiores detalhes sobre as premissas para o cálculo do tamanho da amostra podem ser observados em LimaCosta et al. (2000).

Entre os 1.664 indivíduos selecionados para participar do inquérito de saúde de Bambuí, $1.382(83,1 \%)$ participaram do inquérito sobre saúde bucal (os participantes do inquérito de saúde bucal eram semelhantes à população amostrada em relação ao sexo e faixa etária). Entre os participantes do inquérito de saúde bucal, foram selecionados para o presente trabalho todos os que (1) tinham idade igual ou superior a 18 anos, (2) possuíam pelo menos um dente natural e (3) haviam visitado o dentista pelo menos uma vez na vida. Um grupo de 656 indivíduos preenchia estes critérios. Este número é suficiente para detectar uma odds ratio $\geq 1,50$, com intervalo de confiança $=0,95$, sendo a prevalência da característica entre os usuários de serviços ocasionais $=0,30$ e razão entre usuários regulares/usuários ocasionais = 3:1 (Dean et al., 1994).

Todos os participantes do Projeto Bambuí receberam uma carta explicando os objetivos e procedimentos do estudo e assinaram um consentimento informado. O Projeto Bambuí foi aprovado pelo Comitê de Ética da Fundação Oswaldo Cruz (Lima-Costa et al., 2000).

\section{Coleta de dados}

A coleta de dados foi realizada entre outubro de 1996 e janeiro de 1997. As informações foram obtidas mediante entrevista face a face realizada no domicílio do participante. Seis entrevistadores foram selecionados entre residentes na Cidade de Bambuí que possuíam no mínimo o 2 o grau completo. O treinamento dos entrevistadores foi feito por um dos autores (DLM). Foi utilizado um questionário estruturado, previamente validado e pré-codificado; a entrevista durava em média vinte minutos.

\section{Variáveis do estudo}

Modelo proposto por Andersen \& Newman (1973) foi utilizado como a base conceitual para este estudo. A variável dependente foi o padrão de uso de serviços odontológicos: regular e ocasional. Foram considerados como usuários regulares aqueles que fizeram pelo menos uma visita ao dentista no último ano e que relataram ser a revisão o principal motivo das suas visitas ao dentista (Todd \& Lader, 1991). Os demais foram considerados como usuários ocasionais.

Como variáveis explicativas foram consideradas: (a) de predisposição: sexo (masculino, feminino); faixa etária (18-38, 39-59 $e \geq 60$ anos); escolaridade ( $\leq 3$ anos, $4-7$ anos $e \geq 8$ anos); tipo de tratamento preferido para dente anterior e posterior (extração em pelo menos uma situação, restauração para as duas situações); crença de que ir ao dentista evita cárie e doença na gengiva (sim, não); (b) de facilitação: renda familiar (<2, 2-3,99e $e$ 4,00 salários mínimos); (c) de necessidade: percepção da necessidade atual de tratamento dentário (sim, não).

\section{Análise dos dados}

A análise dos dados foi baseada nos testes do Qui-quadrado e Qui-quadrado para tendências lineares para verificar a existência de associações entre variáveis. Odds ratios e seus intervalos de confiança (método de Woolf) no nível de 0,95 foram utilizadas para estimar as forças das associações entre variáveis (Armitage \& Berry, 1988). O método de regressão logística múltipla foi utilizado para examinar o efeito independente de cada variável (Breslow \& Day, 1987). Sexo, faixa etária, renda familiar ou escolaridade foram considerados, a priori, variáveis de confusão neste estudo. Nos modelos logísticos foram incluídas, além de sexo e faixa etária, as seguintes variáveis: escolaridade ou renda familiar, tipo de tratamento preferido para dente anterior e posterior, acreditar que ir ao dentista evita cárie e doença da gengiva e percepção da necessidade atual de tratamento dentário. Foram construídos dois modelos logísticos: no primeiro, a variável escolaridade foi incluída, mas não a renda familiar; no segundo, a renda familiar foi incluída, mas não a escolaridade. No presente trabalho optou-se por mostrar o modelo que incluía escolaridade, uma vez que esta mostrou associação mais forte com o uso regular de serviços odontológicos do que a renda familiar. No entanto, é importante relatar que a associação com a renda familiar manteve se estatisticamente significante após ajustamento pelas outras variáveis estudadas. Os dados foram analisados utilizandose o software STATA (Stata Corporation, 1997). 


\section{Resultados}

Das 656 pessoas que preenchiam os critérios para inclusão neste estudo, 654 participaram do trabalho. Destes, 161 (24,6\%) foram classificados como usuários regulares e $493(75,4 \%)$ como usuários ocasionais de serviços odontológicos.

A idade média dos participantes foi de 35 anos (variação = 18-89 anos), 48,2\% eram homens e $51,8 \%$ eram mulheres. Cerca da metade dos participantes possuía renda familiar igual ou inferior a 3,99 salários mínimos $(48,8 \%)$ e oito anos ou mais de escolaridade $(45,7 \%)$.

As variáveis de predisposição que apresentaram associações positivas e estatisticamente significantes com a visita regular ao dentista na análise univariada foram as seguintes: maior escolaridade $(\mathrm{OR}=4,14$; IC $95 \%=1,44-11,92 \mathrm{e}$ OR $=15,16$; IC $95 \%=5,43-42,34$ para aqueles com $4-7$ e $\geq 8$ anos de escolaridade, respectivamente), ter a restauração como tratamento preferido para dente anterior e posterior $(\mathrm{OR}=$ 6,14 ; IC $95 \%=3,23-11,65$ ) e acreditar que ir ao dentista evita a cárie e doença na gengiva $(\mathrm{OR}=$ 1,62; IC $95 \%=1,13-2,32$ ). A faixa etária de $60 \mathrm{ou}$ mais anos esteve negativamente associada à visita regular ao dentista $(\mathrm{OR}=0,30$; $\mathrm{IC} 95 \%=$ 0,10-0,86) (Tabela 1).

A distribuição da visita regular ao dentista, segundo as variáveis de facilitação e necessidade está apresentada na Tabela 2. Renda familiar $\geq 4,00$ salários mínimos (OR = 4,48; IC 95\% = 2,24-8,98) e não perceber necessidade atual de

Tabela 1

Distribuição percentual da visita regular ao dentista, segundo as variáveis de predisposição. Bambuí, 1996-97.

\begin{tabular}{|c|c|c|c|c|}
\hline \multirow[t]{2}{*}{ Variáveis de predisposição } & \multicolumn{2}{|c|}{ Visita regular ao dentista } & \multirow[t]{2}{*}{ Total } & \multirow[t]{2}{*}{ OR (IC 95\%) } \\
\hline & $\underset{(n=161) \%}{\operatorname{Sim}}$ & $\begin{array}{c}\text { Não } \\
(n=493) \%\end{array}$ & & \\
\hline \multicolumn{5}{|l|}{ Sócio-demográficas } \\
\hline \multicolumn{5}{|l|}{ Sexo } \\
\hline Masculino & 44,7 & 49,3 & 48,2 & 1,00 \\
\hline Feminino & 55,3 & 50,7 & 51,8 & $1,20(0,84-1,72)$ \\
\hline \multicolumn{5}{|c|}{$p=0,314$} \\
\hline \multicolumn{5}{|l|}{ Faixa etária (em anos) } \\
\hline $18-38$ & 71,4 & 62,9 & 65,0 & 1,00 \\
\hline $39-59$ & 26,1 & 29,8 & 28,9 & $0,77(0,51-1,15)$ \\
\hline$\geq 60$ & 2,5 & 7,3 & 6,1 & $0,30(0,10-0,86)$ \\
\hline \multicolumn{5}{|c|}{$p^{\prime}=0,015$} \\
\hline \multicolumn{5}{|l|}{ Escolaridade (em anos) } \\
\hline$\leq 3$ & 2,5 & 18,9 & 14,8 & 1,00 \\
\hline $4-7$ & 24,2 & 44,4 & 39,5 & $4,14(1,44-11,92)$ \\
\hline$\geq 8$ & 73,3 & 36,7 & 45,7 & $15,16(5,43-42,34)$ \\
\hline \multicolumn{5}{|c|}{$p^{\prime}=0,000$} \\
\hline \multicolumn{5}{|l|}{ Atitudes e opiniões } \\
\hline \multicolumn{5}{|l|}{$\begin{array}{l}\text { Tratamento preferido para } \\
\text { o dente anterior e posterior }\end{array}$} \\
\hline Extração e Extração/Restauração & 6,8 & 1,0 & 25,1 & 1,00 \\
\hline \multirow[t]{2}{*}{ Restauração } & 93,2 & 69,0 & 74,9 & $6,14(3,23-11,65)$ \\
\hline & \multicolumn{2}{|c|}{$p=0,000$} & & \\
\hline \multicolumn{5}{|l|}{$\begin{array}{l}\text { Acreditar que ir ao dentista evita } \\
\text { cárie e doença na gengiva }\end{array}$} \\
\hline Não & 43,5 & 55,4 & 52,4 & 1,00 \\
\hline Sim & 54,6 & 43,0 & 45,9 & $1,62(1,13-2,32)$ \\
\hline \multirow[t]{2}{*}{ Não sabe } & 1,9 & 1,6 & 1,7 & - \\
\hline & \multicolumn{2}{|c|}{$p=0,009$} & & \\
\hline
\end{tabular}

$\mathrm{p}=$ teste do Qui quadrado

$\mathrm{P}^{\prime}=$ teste do Qui quadrado para tendências lineares

odds ratio $(\mathrm{IC} 95 \%)=$ odds ratio bruta e intervalo de confiança de $95 \%$ 
tratamento dentário $(\mathrm{OR}=5,22$; IC $95 \%=3,55$ 7,70 ) apresentaram associações positivas e estatisticamente significantes com a visita regular ao dentista.

Na Tabela 3 estão apresentados os resultados finais da análise multivariada das variáveis associadas à visita regular ao dentista. As seguintes variáveis apresentaram associações independentes com as visitas regulares ao dentista: maior escolaridade $(\mathrm{OR}=9,90$; IC 95\% = $2,90-33,77$ e OR $=3,87$; IC $95 \%=1,11-13,5$ para aqueles com $\geq 8$ e 4-7 anos de escolaridade, respectivamente); ter a restauração como tratamento preferido para o dente anterior e posterior $(\mathrm{OR}=4,91$; IC $95 \%=2,23-10,79)$, acreditar que ir ao dentista evita cárie e doença na gengiva $(\mathrm{OR}=1,73$; IC $95 \%=1,13-2,65)$ e não perceber necessidade atual de tratamento dentário $(\mathrm{OR}=4,87$; IC 95\% 3,17-7,49).

\section{Discussão}

As variáveis de predisposição, facilitação e necessidade contribuíram para explicar o padrão de uso de serviços odontológicos na comunidade estudada. As associações encontradas no presente trabalho estão consistentes com o observado em outros estudos (Gilbert et al., 1993, 1997; Swank et al., 1986) que utilizaram o modelo proposto por Andersen \& Newman (1973). As variáveis que se mostraram independentemente associadas ao uso regular de serviços odontológicos foram: maior escolaridade (4-7 anos e $\geq 8$ anos), tratamento restaurador preferido para dente anterior e posterior, não ter necessidade atual de tratamento odontológico e acreditar que ir ao dentista evita cárie e doença na gengiva.

De acordo com trabalhos recentes (Gilbert et al., 1993, 1997), mas ao contrário de resultados obtidos em estudos mais antigos (Eddie \& Davies, 1985), as variáveis demográficas de predisposição (idade e sexo) não estiveram associadas ao padrão de uso de serviços odontológicos. A forte associação observada no presente trabalho entre escolaridade, outra variável de predisposição tradicionalmente estudada, e uso regular de serviços confirma achados de estudos anteriores a este (Gilbert et al., 1993; Swank et al., 1986).

Neste trabalho foram incluídas as variáveis tratamento preferido (restaurador ou extração) e crença de que ir ao dentista evita cárie e doen-

Tabela 2

Distribuição percentual da visita regular ao dentista, segundo as variáveis de facilitação e necessidade. Bambuí, 1996-97.

\begin{tabular}{|c|c|c|c|c|}
\hline \multirow[t]{2}{*}{ Variáveis de predisposição } & \multicolumn{2}{|c|}{ Visita regular ao dentista } & \multirow[t]{2}{*}{ Total } & \multirow[t]{2}{*}{ OR (IC 95\%) } \\
\hline & $\operatorname{Sim}_{(\mathrm{n}=161) \%}$ & $\begin{array}{c}\text { Não } \\
(n=493) \%\end{array}$ & & \\
\hline \multicolumn{5}{|l|}{ Facilitação } \\
\hline \multicolumn{5}{|l|}{$\begin{array}{l}\text { Renda familiar (em salários } \\
\text { mínimos da época)* }\end{array}$} \\
\hline$<2$ & 6,2 & 17,0 & 14,4 & 1,00 \\
\hline $2-3,99$ & 22,4 & 39,4 & 35,2 & $1,56(0,74-3,29)$ \\
\hline$\geq 4,00$ & 68,9 & 42,2 & 48,8 & $4,48(2,24-8,98)$ \\
\hline Sem informação & 2,5 & 1,4 & 1,6 & - \\
\hline \multicolumn{5}{|c|}{$p^{\prime}=0,000$} \\
\hline \multicolumn{5}{|l|}{ Necessidade } \\
\hline \multicolumn{5}{|c|}{$\begin{array}{l}\text { Percepção de necessidade atual } \\
\text { de tratamento dentário }\end{array}$} \\
\hline Sim & 36,0 & 73,7 & 64,4 & 1,00 \\
\hline Não & 59,6 & 23,3 & 32,2 & $5,22(3,55-7,70)$ \\
\hline Não sabe & 4,4 & 3,0 & 3,4 & - \\
\hline \multicolumn{5}{|c|}{$p=0,000$} \\
\hline
\end{tabular}

$p=$ teste do Qui quadrado

$\mathrm{P}^{\prime}=$ teste do Qui quadrado para tendências lineares

OR (IC 95\%) = odds ratio bruta e intervalo de confiança de $95 \%$

* Salário mínimo da época $(\mathrm{R} \$ 120,00)$ 
Tabela 3

Resultados estatisticamente significantes obtidos na análise multivariada dos fatores associados ao uso regular de serviços odontológicos. Bambuí, 1996-97.

\begin{tabular}{ll}
\hline Variáveis & OR ajustada (IC 95\%) \\
\hline $\begin{array}{l}\text { Predisposição } \\
\text { Escolaridade (em anos) }\end{array}$ \\
$\quad 3$ & 1,00 \\
$4-7$ & $3,87(1,11-13,51)$ \\
$\geq 8$ & $9,90(2,90-33,77)$ \\
Tratamento preferido para & \\
O dente anterior e posterior & \\
$\quad$ Extração e Extração/Restauração & 1,00 \\
Restauração & $4,91(2,23-10,79)$ \\
Acreditar que ir ao dentista evita & \\
cárie e doença na gengiva & \\
Não & 1,00 \\
Sim & $1,73(1,13-2,65)$ \\
Necessidade & \\
Percepção de necessidade atual & \\
de tratamento dentário & \\
Sim & 1,00 \\
Não & $4,87(3,17-7,49)$ \\
\hline OR ajustada (IC $95 \%)$ & $95 \%$ ajustados
\end{tabular}

OR ajustada (IC 95\%) = odds ratio e intervalo de confiança de $95 \%$ ajustados por sexo, faixa etária e todas as variáveis listadas na tabela (621 indivíduos participaram do modelo final). padrão de uso de serviços odontológicos; esta forte associação permaneceu após ajustamentos por variáveis de confusão. A forte associação encontrada entre escolaridade, tipo de tratamento preferido e o padrão de uso de serviços leva-nos a sugerir que a limitação financeira não deve ser uma barreira suficiente para determinar o uso de serviços odontológicos. De acordo com Gilbert (1995), o custo não parece ser a principal barreira para o uso do serviços odontológicos e a remoção desta barreira não necessariamente igualaria o uso de serviços entre as pessoas com diferentes níveis sócio-econômicos (Locker et al., 1991). No entanto, a interpretação destes achados deve ser feita com muito cuidado. É importante notar que a variável escolaridade é altamente correlacionada com a variável renda, e a maior sensibilidade da primeira deve-se ao fato de escolaridade ser um indicador mais amplo, não significando de forma alguma que renda não seja um importante preditor de uso de serviços odontológicos.

A variável de necessidade neste estudo (percepção da necessidade atual de tratamento dentário) também foi um importante preditor do padrão de uso de serviços odontológicos. Porém, ao contrário do esperado, as pessoas que responderam não necessitar de tratamento apresentaram maior tendência a visitar regularmente o dentista. Este resultado confirma os achados de Tennstedt et al. (1994), demonstrando que aqueles que são usuários regulares têm menor necessidade de tratamento odontológico.

Apesar do rigor metodológico seguido durante a realização deste estudo, algumas limitações devem ser discutidas. A utilização de entrevista como forma de coleta de dados pode introduzir um viés de memória no estudo. Também não foi possível incluir todas as potenciais barreiras ao uso regular de serviços. Por exemplo, alguns estudos (Douglass \& Cole, 1979; Kreisberg \& Treiman, 1962) têm mostrado que a ansiedade e o medo da dor constituem uma barreira para o uso de serviços odontológicos e este achado tem-se mostrado consistente entre pessoas de diversos níveis sócioeconômicos. No presente trabalho esta variável não foi levada em consideração. Por outro lado, a similaridade dos nossos resultados com outros estudos citados anteriormente sustentam a validade das respostas obtidas no presente trabalho. Finalmente, é importante ressaltar que a variável dependente foi criteriosamente construída. O tempo decorrido desde a última visita ao dentista foi utilizado para comprovação da resposta à pergunta sobre padrão de uso de serviços, regular ou ocasional. 
Este estudo representou um esforço para explicar o padrão de uso de serviços odontológicos em uma população adulta utilizando-se o modelo proposto por Andersen \& Newman (1973) e, conseqüentemente, fornecer dados para o planejamento de serviços de saúde bucal. A revisão da literatura mostrou que apenas em um estudo foram examinados os fatores associados ao uso de serviços odontológicos na população brasileira. É importante entender por que algumas pessoas esperam até que seus dentes necessitem de extenso tratamento para procurar cuidados odontológicos, enquanto outras procuram regularmente esses serviços. O uso regular de serviços odontológicos pode acarretar uma redução nos gastos com tratamento, devido à intervenção na fase inicial da doença, quando a destruição do elemento dentário é extremamente menor (Newman \& Gift, 1992).

\section{Agradecimentos}

Este trabalho foi financiado pela Financiadora de Estudos e Projetos (FINEP). A Coordenação de Aperfeiçoamento de Pessoal de Nível Superior (CAPES) e o Conselho Nacional de Desenvolvimento Científico e Tecnológico (CNPq) concederam bolsas para alguns dos autores (DLM, HLG e MFFCL). Nós gostaríamos também de agradecer ao Dr. Marco Aurélio Peres, Universidade Federal de Santa Catarina, pela revisão crítica do manuscrito. Sem a adesão da população de Bambuí, este estudo não teria sido viável.
Em resumo, os resultados deste trabalho, baseados no modelo proposto por Andersen \& Newman (1973), mostraram que também em Bambuí o uso regular de serviços odontológicos pode ser explicado por características de predisposição, facilitação e necessidade. Vários trabalhos têm examinado os fatores associados ao uso de serviços odontológicos (Gilbert et al., 1993, 1997; Locker \& Clarke, 1999; Ryan et al., 1996; Swank et al., 1986; Tennstedt et al., 1994); entretanto, a influência do tipo de tratamento preferido para os dentes anteriores e posteriores tem sido pouco estudada (Gilbert et al., 1997). Esta variável, juntamente com nível de escolaridade e percepção de necessidade atual de tratamento dentário foram as mais fortemente associadas ao uso regular de serviços odontológicos na comunidade estudada.

\section{Referências}

ANDERSEN, R. \& NEWMAN, J. F., 1973. Societal and individual determinants of medical care utilization in the United States. Milbank Memorial Fund Quartely, 51:95-124.

ARMITAGE, P. \& BERRY, G., 1988. Statistical Methods in Medical Research. 2nd Ed. London: Blackwell Scientific Publications.

BRESLOW, N. E. \& DAY, N. E., 1987. Statistical Methods in Cancer Research: The Analysis of Cohort Studies. Lyon: International Agency for Research on Cancer.

BROWN, L. J. \& LAZAR, V., 1999. Dental care utilization: How satured is the patient market? Journal American Dental Association, 130:573-580.

CESAR, C. L. G.; NARVAI, P. C.; FIGUEIREDO, G. M. \& GATTÁS, V. L., 1995. Utilização de serviços odontológicos em municípios da Grande São Paulo, 1989-1990. In: III Congresso Brasileiro de Epidemiologia, Resumos, p. 28, Salvador: ABRASCO.

CHERRY-PEPPERS, G.; SINKFORD, J. C.; NEWMAN, E. S.; SANDERS, C. F .\& KNIGHT, R. S., 1995. Primary oral health care in black americans: An asssessment of current status and future needs. Journal of the National Medical Association, 87: 136-140.

CHISICK, M. C.; POINDEXTER, F. R. \& YORK, A. K., 1997. Factors influencing perceived need for dental care by active duty U.S. military personnel. Military Medicine,162:586-589. 
DEAN, A. G.; DEAN, J. A.; COULOMBIER, D.; BRENDEL, K. A.; SMITH, D. C.; BURTON, A. H.; DICKER, R. C.; SULLIVEN, K.; TAGAN, R. F. \& ARNET, T. G., 1994. Epi Info, version 6.0: A Word Processing Database and Statistics Program for Epidemiology on Microcomputers. Atlanta: Centers for Disease Control and Prevention.

DOUGLASS, C. W. \& COLE, K. O., 1979. Utilization of dental services in the United States. Journal of Dental Education, 43:223-238.

EDDIE, S. \& DAVIES, J. A., 1985. The effect of social class on attendance frequency and dental treatment received in the general dental service in Scotland. British Dental Journal, 159:370-372.

GILBERT, H. G., 1995. Acess to and patterns of use of oral health care among elderly veterans. Medical Care, 33:78-89.

GILBERT, H. G.; BRANCH, L. G. \& LONGMATE, J., 1993. Dental care use by U.S. veterans elegible for VA care. Social Science and Medicine, 33:361-370.

GILBERT, H. G.; DUNCAN, R. P.; HEFT, M. W. \& COWARD, R. T., 1997. Dental health attitudes among dentate black and white adults. Medical Care, 35: 255-271.

GREMBOWSKI, D.; ANDERSEN, R. M. \& CHEN, M. S., 1989. A public health model for dental care process. Medical Care Review, 46:439-496.

IBGE (Fundação Instituto Brasileiro de Geografia e Estatística), 1992. Anuário Estatístico do Brasil. Rio de Janeiro: IBGE.

HONKALA, E.; KUUSELA, S.; RIMPELÄ, A.; RIMPELÄ, M. \& JOKELA, J., 1997. Dental services utilization between 1977 and 1995 by Finnish adolescents of different socioeconomic levels. Community Dentistry and Oral Epidemiology, 25:385-390.

KREISBERG, L. \& TREIMAN, B. R., 1962. Preventive utilization of dentists' services among teenagers. Journal of the American College of Dentists, 29:2845.

LIMA-COSTA, M. F. F.; UCHOA, E.; GUERRA, H. L.; FIRMO, J. A. O.; VIDIGAL, P. G. \& BARRETO, S. M., 2000. Methodological approach for a population based cohort study of the elderly in Brazil and preliminary results: The Bambuí Health and Ageing Study (BHAS). Revista de Saúde Pública, 34: 126-135.
LOCKER, D. \& CLARKE, M., 1999. Geografic variations in dental services provided to older adults in Ontario, Canada. Community Dentistry and Oral Epidemiology, 27:275-282.

LOCKER, D.; LEAKE, J. L.; LEE, J.; MAIN, P. A.; HICKS, T. \& HAMILTON, M., 1991. Utilization of dental services by older adults in four Ontario communities. Journal of the Canadian Dental Association, 57:879-886.

MARCOS, B., 2000. Ensino Odontológico - novos cursos, mais profissionais e menos demanda da população. Jornal do Conselho Regional de Odontologia, Belo Horizonte, 107:8-9.

NEWMAN, J. F. \& GIFT, H. C., 1992. Regular pattern of preventive dental service - A measure of acess. Social Science and Medicine, 35:997-1001.

PETERSEN, P. E. \& PEDERSEN, K. M., 1984. Socioeconomic demand model for dental visits. Community Dent Oral Epidemiol, 12:361-365.

PREFEITURA MUNICIPAL DE BAMBUÍ, 1997. Plano Municipal de Saúde. Bambuí: Prefeitura Municipal.

RYAN, S. A.; MILLSTEIN, S. G.; GREENE, B. \& IRWIN C. E., 1996. Utilization of ambulatory health services by urban adolescents. Journal of Adolescent Health, 18:192-202.

SCHWARZ, E., 1996. Changes in demand for dental care among Danish adults, 1975-90. Acta Odontologica Scandinavia, 54:36-43.

STATA CORPORATION, 1997. Stata Statistical Software: Release 6.0. College Stations, Stata Corporation.

SWANK, M. E.; VERNON, S. W. \& LAIRSON, D. R., 1986. Patterns of preventive dental behavior. Public Health Reports, 101:175-84.

TENNSTEDT, S. L.; BRAMBILLA, D. L.; JETTE, A. M. \& McGUIRE, S. M., 1994. Understanding dental service use by older adults sociobehavioral factors vs need. Journal of Public Health Dentistry, 54: 211-219.

TODD, J. E. \& LADER, D., 1991. Adult Dental Health 1988: United Kingdom. London: Her Majesty's Stationary Office. 